\title{
Beta spectrum shape measurements using a multi-wire drift chamber and a plastic scintillator
}

\author{
L. De Keukeleere, ${ }^{a, *}$ D. Rozpedzik, ${ }^{b}$ K. Bodek, ${ }^{b}$ L. Hayen, ${ }^{c}$ K. Lojek, ${ }^{b}$ M. Perkowski, ${ }^{a, b}$ \\ N. Severijns ${ }^{a}$ and S. Vanlangendonck ${ }^{a}$ \\ ${ }^{a}$ Institute of Nuclear and Radiation Physics, KU Leuven, \\ B-3001 Leuven,Belgium \\ ${ }^{b}$ Marian Smoluchowski Institute of Physics, Jagiellonian University, \\ 30348 Krakow, Poland \\ ${ }^{c}$ Department of Physics, North Carolina State Univeristy, \\ Raleigh, NC 27695-8202, USA
}

E-mail: lennert.dekeukeleere@kuleuven.be

Spectrum shape measurements in nuclear $\beta$ decay are a versatile observable. They can be used to test physics beyond the Standard Model with results being complementary to high energy collider experiments. In addition, the $\beta$ spectrum shape is a useful tool to probe Standard Model effects. One of those effects is called Weak Magnetism and is induced by QCD interactions between quarks in the nucleon.

In order to study effects on the order of $10^{-3}-10^{-2}$ in the $\beta$ spectrum shape, a new prototype spectrometer, named miniBETA, was designed and built. It consists of a 3D low-pressure gas tracker, i.e. a multi-wire drift chamber with hexagonal cells, and a plastic scintillator for triggering the data acquisition and recording the $\beta$ particle energy.

Results of the miniBETA spectrometer characterization, supported by Monte Carlo simulations in Geant 4 and Garfield++, are reported here. In addition, the preliminary results from $\beta$ spectrum shape measurements on the allowed Gamow-Teller transition ${ }^{114} \mathrm{In} \rightarrow{ }^{114} \mathrm{Sn}$ are presented, including an extraction of the Weak Magnetism form factor.

\footnotetext{
*** Particles and Nuclei International Conference - PANIC2021 ***

*** 5 - 10 September, $2021 * * *$

*** Online ***

* Speaker
} 


\section{Introduction}

Beyond Standard Model (BSM) theories are probed in different types of experiments. In collider experiments, i.e. at LHC, one tries to directly produce new exotic bosons in high-energy proton - proton collisions. Another way to test BSM theories, is by studying low-energy observables. One then searches for the small effects the exotic bosons would induce on these observables. The shape of the $\beta$ spectrum, which is the topic of this research, is sensitive to two exotic currents, scalar and tensor, both prohibited in the Standard Model (SM) weak interaction. For allowed $\beta$ transitions, these currents would introduce a correction term in the spectrum, called the Fierz term $b_{\text {Fierz }}$, which is inversely proportional to the $\beta$ particle energy[1].

Reaching the $0.5 \%$ precision level, higher-order SM effects cannot be neglected. An important higherorder effect is induced by the strong interaction and the fact that the decaying quark is not a free particle but embedded in the nucleon. This effect is dominated by the so-called Weak Magnetism (WM) term (e.g. [2]). The lack in knowledge of WM has already limited the attainable precision of several of the recent experiments or constituted a major contribution to their systematic error bar [3]. This is especially important for searches of tensor currents, which typically use pure Gamow-Teller transitions in order to optimize sensitivity (WM is absent in pure Fermi transitions). Recently, an update was made of existing experimental knowledge for the WM form factor $b_{\mathrm{WM}}$, combined with shell-model based calculations to gain further insight in its nuclear structure and/or mass dependence[4]. However, experimental knowledge is limited to nuclei with mass number up to $A=61$. Hence, a measurement of WM in the higher mass region could serve as a benchmark for nuclear shell-model calculations. In addition, for some particular transitions a measurement of WM can provide a good test for the Conserved Vector Current hypothesis (CVC) [2]. Furthermore, the knowledge of WM for high-mass neutron-rich nuclei is important in the analysis of reactor anti-neutrino experiments[5].

When taking both the Beyond Standard Model Fierz term and the Standard Model WM term into account, the $\beta$ spectrum shape for an allowed Gamow-Teller decay can be written as:

$$
W\left(E_{e}\right) d E_{e}=\frac{F\left( \pm Z, E_{e}\right)}{2 \pi^{3}} p_{e} E_{e}\left(E_{0}-E_{e}\right)^{2} d E_{e} \xi\left(1+b_{\mathrm{Fierz}} \frac{m_{e}}{E_{e}} \pm \frac{4}{3} \frac{E_{e}}{M_{n}} \frac{b_{\mathrm{WM}}}{A c}\right),
$$

with $F\left( \pm Z, E_{e}\right)$ the Fermi function, which includes the dominant Coulomb correction, $\xi=\left|C_{A}\right|^{2}+\left|C_{A}^{\prime}\right|^{2}+$ $\left|C_{T}\right|^{2}+\left|C_{T}^{\prime}\right|^{2}$ a factor including the axial and tensor couplings, $E_{e}$ and $p_{e}$ the total energy and momentum of the $\beta$ particle, $E_{0}$ the maximum total electron energy, $m_{e}$ the electron mass, $M_{n}$ the nucleon mass, and $c=C_{A} M_{G T}$ the Gamow-Teller form factor. The upper (lower) sign refers to $\beta^{-}\left(\beta^{+}\right)$decay, and $A$ and $Z$ are the mass number and charge of the daughter nucleus, respectively.

In order to improve existing constraints on BSM currents, a precision of $10^{-3}$ is required when determining $b_{\text {Fierz }}$ [1]. Based on previously measured values of $b_{\mathrm{WM}}$ a spectrum shape distortion at the level of $10^{-3}-10^{-2}$ is expected, due to WM[4]. With this in mind, the current research attempts to measure the pure Gamow-Teller ${ }^{114} \mathrm{In} \rightarrow{ }^{114} \mathrm{Sn}$ spectrum shape at the $10^{-3}$ to $10^{-2}$ precision level.

\section{Apparatus}

The miniBETA apparatus (Fig. 1, panel A) is a combination of a $\beta$ particle energy detector and a low-energy charged particle gas tracker. The former comprises a plastic scintillator disk with a thickness of $3 \mathrm{~cm}$, optically coupled to a light guide disk on which 4 PMT's are stacked (Fig. 1, panel B). The latter is a honeycomb-like multi-wire drift chamber (MWDC) with anode wires in the centre at high voltages around $2000 \mathrm{~V}$, and grounded cathode wires at the corners (Fig. 1, panel C). The gas medium is a mixture of helium and isobutane. Based on performance studies, it was decided to use a ratio of $70 \%$ helium to $30 \%$ isobutane at a pressure of 600 mbar. The purpose of the MWDC is to identify events that distort the spectrum shape, e.g. electrons back-scattering from the scintillator surface or cosmic muons flying through the experimental setup. In addition to event pattern recognition, the setup allows for several filtering and calibration methods. For example, by requiring coincidence between the scintillator and drift chamber, noise and gamma particles 
are filtered. Furthermore, in order to correct for non-uniform light propagation in scintillator and light guide, tracking conversion electrons from a ${ }^{207} \mathrm{Bi}$ calibration source, located next to the $\beta$ source, enables the real-time generation of a 2D-detector surface gain map (see Sec. 3).

The 3D-track reconstruction in the MWDC occurs in two steps. First, the track is reconstructed in the plane perpendicular to the wires (Fig. 1, panel C), using the relation between trigger delay and the track's distance of closest approach $R$ to the anode wire (top of panel C). A Legendre tracking method is devised to find a straight track, minimizing the difference between $d_{i}$ and $R_{i}$ (bottom of panel C). Second, the track is reconstructed in the plane parallel to the wires (Fig. 1, panel D). Here, a model of charge division is employed to infer the position along each wire traversed by the particle (top of panel D). A simple straight line fit then attempts to reconstruct the track in this second plane (bottom of panel D). Track reconstruction precisions of $\sim 1 \mathrm{~mm}$ and $\sim 10 \mathrm{~mm}$ were obtained in the respective projection planes.

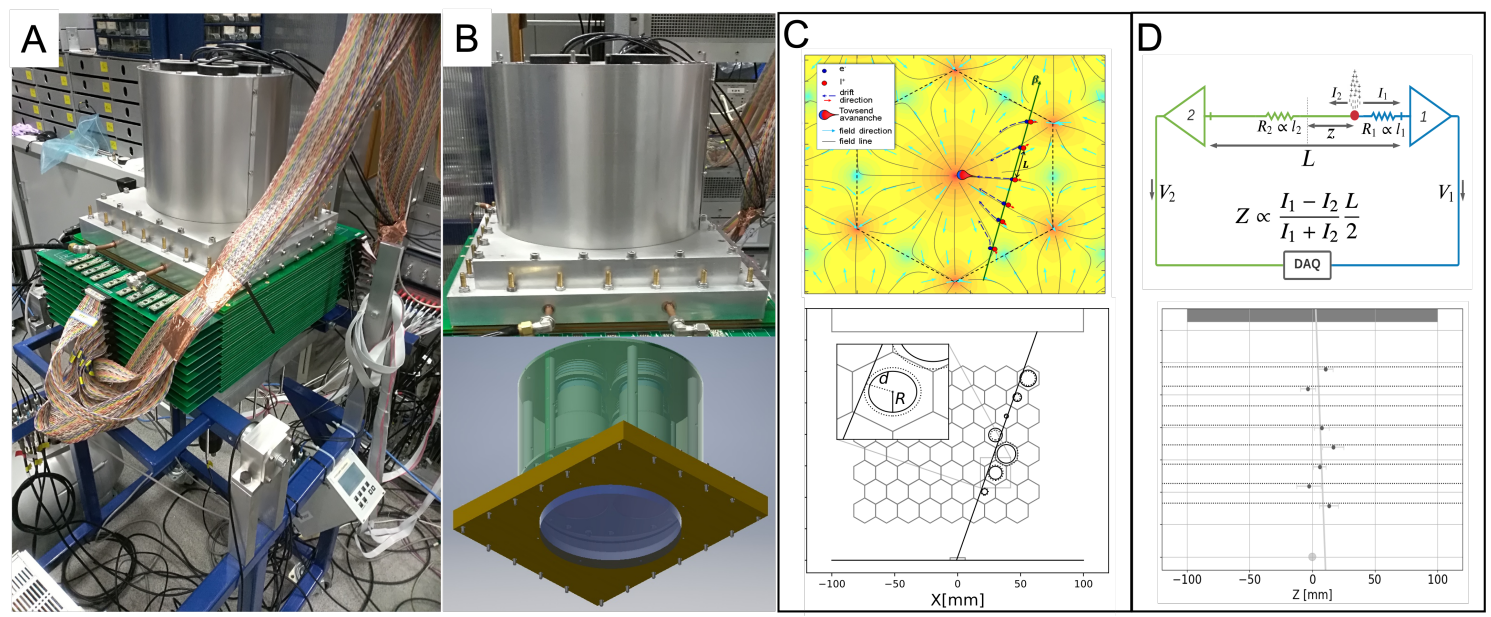

Figure 1: The miniBETA apparatus. Panel A: a picture of the full setup, with the MWDC (visible as green PCB boards with the front-end electronics) and the PMT housing on top. Panel B: (top) external view of the energy detector with the aluminium end cap and PMT housing, and (bottom) 3D CAD scheme of the detector seen from below, showing the cylindrical plastic scintillator in blue, the end cap in brown and the inside of the PMT housing in green. Panel C: (top) a hexagonal cell in the plane perpendicular to the wires, with the HV anode wire in the centre and the grounded cathode wires at the corners. The colours and lines show the electric field magnitude and shape, respectively. The ionization electrons drift towards the centre along the electric field lines, leaving behind a signal on the anode wire. From the signal delay the distance $R$ at which the track passes can be inferred and used to reconstruct the 2D-track (bottom). Panel D: (top) the charge division model, used for the simple straight line fit in the plane parallel to the wires(see bottom picture).

\section{Methods}

First of all, the track reconstruction algorithm is responsible for recognizing patterns and identifying different event origins. As demonstrated in the left panel of Fig. 2, the applied data analysis methods are able to distinguish cosmic muons, ${ }^{207} \mathrm{Bi}$ calibration electrons, ${ }^{114} \mathrm{In} \beta$ particles and backscattered electrons. The recognition efficiency was tested with MC simulations [6] and found to be extremely good, giving rise to $>99 \%$ clean ${ }^{207} \mathrm{Bi}$ conversion and ${ }^{114} \mathrm{In} \beta$ spectra. In addition, the algorithm reduces the number of backscatter events by $50 \%$.

Secondly, the 3D-track reconstruction is employed to extrapolate the detector entry point. This information is crucial, since the light collection efficiency is position dependent. By dividing the scintillator surface in a grid of $1 \times 1 \mathrm{~cm}^{2}$ squares and by fitting the ${ }^{207} \mathrm{Bi}$ conversion electron energy spectrum for the set of events entering the detector in the selected area (middle panel of Fig. 2), a 2D map of the energy calibration parameters can be constructed (right panel). This, in turn, can be used to reconstruct the $\beta$ spectrum shape. 

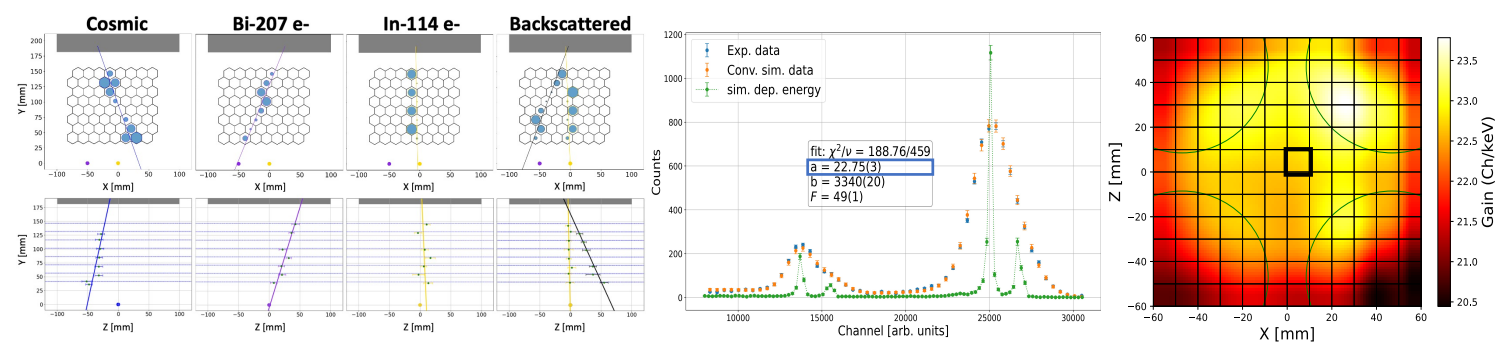

Figure 2: Left panel: Different event origins, recognized by the track algorithm. Middle and right panel: A visual description of the 2D position-dependent scintillator energy calibration procedure. See the text for more details.

\section{Results and Outlook}

As a first proof of principle, the total experimental and simulated ${ }^{207} \mathrm{Bi}$ and ${ }^{114} \mathrm{In} \beta$ spectra, with $b_{\text {Fierz }}$ and $b_{\mathrm{WM}}$ put to zero, were compared (Fig. 3). The experimental spectra were found to be reproduced by simulation at the $10^{-2}$-level. In the Bi-spectrum both the high intensity $500 \mathrm{keV}$ (Channel 12000) and $1000 \mathrm{keV}$ (Channel 26000) peaks, as well as the low intensity coincident transitions (the right shoulder of the $1 \mathrm{MeV}$ bump) and 1.7 MeV (Channel 40000) peak were well matched. The $\beta$ spectrum comparison exhibits a $10^{-2}$-level slope in the fiducial energy region, i.e. $700-1600 \mathrm{keV}$, which could be due to the WM effect.

To test this claim, the same simulation parameters were used to simulate the detector response to electron energies

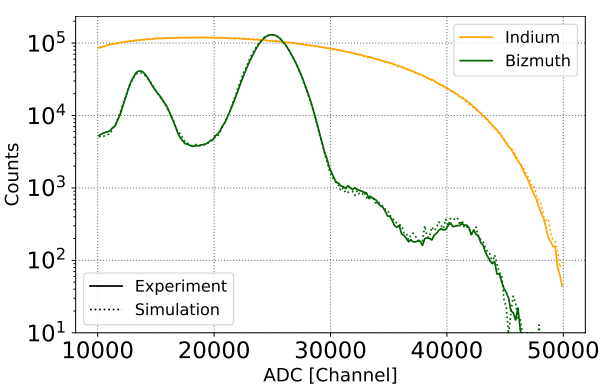

Figure 3: A comparison of the recorded experimental and simulated ${ }^{207} \mathrm{Bi}$ conversion spectra and ${ }^{114}$ In $\beta$ spectra.. ranging from 300 to $2000 \mathrm{keV}$. The WM form factor was then fitted by sampling the theoretical $\beta$ spectrum shape [7] for varying $b_{\mathrm{WM}}$ values, meanwhile assessing the match with the experimental data. The preliminary result of this procedure is presented in Fig. 4. The fit reveals a $b_{\mathrm{WM}}=9.2 \pm 1.2$, which lies within the region of previous measurements on low-mass nuclei.

However, the quoted error bar is merely statistical. A thorough systematic error analysis is currently ongoing.
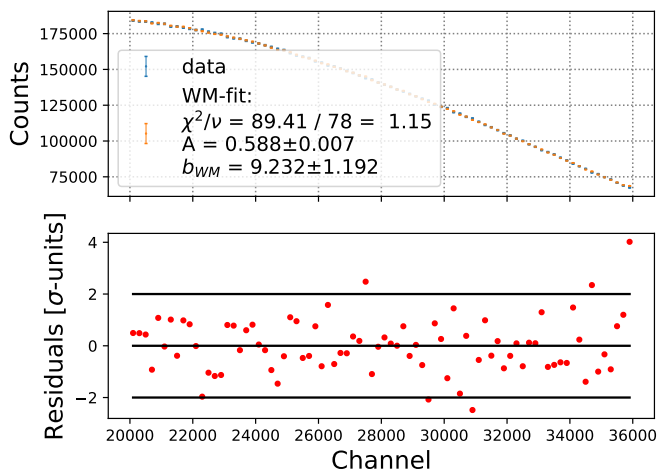

Figure 4: The preliminary WM-fit result for the ${ }^{114}$ In $\beta$ spectrum. See the text for more details.

\section{References}

[1] M. González-Alonso et al., Prog. in Part. and Nucl. Phys. 104, 165-223 (2019).

[2] F. P. Calaprice and B. R. Holstein: Nucl. Phys. A 276 (1976) 301.

[3] F. Wauters et al., Phys. Rev. C 82 (2010) 055502.

[4] N. Severijns et al., submitted to Phys. Rev. C.

[5] A. C. Hayes and P. Vogel, Ann. Rev. of Nucl. and Part. Sc. 66, 219-244 (2016).

[6] L. De Keukeleere et al., Nucl. Instr. and Meth. A 935, 121-134 (2019)

[7] L. Hayen et al., Rev. Mod. Phys. 90, 015008 (2018). 